would fall. A line of wave-length I.037 was recorded, and it was at first thought that this might correspond to the $L a$ radiations of eka-cæsium; but this was apparently not the case, since no corresponding $L B$ line could be detected.

The optical spectrum of a sample of potassium chloride, reduced from some of the chlorplatinate, was also photographed by Messrs. Hilger, but the only impurities we were able to identify were traces of sodium and calcium. It would appear, therefore, that eka-cæsium does not exist in the waters of the Dead Sea. Consideration of its position in the Periodic Table, between radon and radium, would suggest that eka-cæsium may itself be radio-active and short-lived, which latter property might render its detection extremely difficult.

A search was likewise made for the presence of eka-iodine (element 85 ) on the assumption that its silver salt would more be insoluble in water than silver chloride. The latter was fractionally precipitated and exposed to X-ray examination, but without result.

Municipal Technical School, Birmingham, May $\mathbf{~}$.

\section{Influence of Electrical Field on the Adsorption of Neutral Molecules.}

NoT only the adsorption of ions, but also the adsorption of undissociated molecules, depends on the direction and strength of the electrical field at the interface between the adsorbing body and the solution. This circumstance is illustrated by the electrocapillary curves which are obtained if a solution in contact with mercury contains organic substances.

According to Gouy, at a sufficient distance from the maximum the lowering of the interfacial tension by the organic substance vanishes, which means that

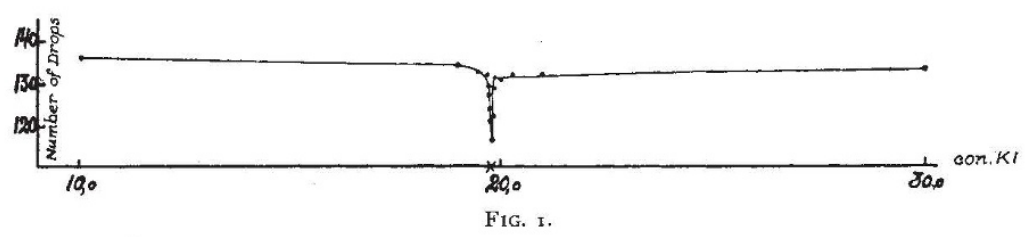
pected. surface. concentration of silver ions in the same way as in the case of a silver electrode, the point at which concentrations of iodine ions and silver ions are equal corresponds for silver iodide to the maximum point of the electrocapillary curve, and near to it a maximum of adsorption for neutral molecules should be ex-

The method adopted for the measurements was the following: to 20 c.c. of an acidified $0.5 \mathrm{~N}$ solution of silver nitrate containing a definite quantity of caprylic acid, a similar solution of potassium iodide was added in small portions. After each addition the concentration of caprylic acid in the solution was determined by its surface tension, measured with a stalagmometer. The data obtained in one series of experiments are plotted on Fig. I. In the proximity to the point of neutralisation a sharp minimum in the number of drops is observed, which corresponds to a minimum value of caprylic acid concentration in the solution, i.e. to its maximum adsorption by the precipitate. The maximum point is somewhat displaced in the direction of an excess of potassium iodide, just as in the case of the adsorption on a mercury

We intend to apply this method to the study of electrocapillary curves of solid metals.

Karpow Chemical Institute,

A. FRUMKIN.

A. Obrutshewa. Moscow, March 18.

\section{Infra-red Emission from Gaseous Explosions.}

THE catalytic effect of water and other substances on the speed of explosions of mixtures of carbon monoxide and oxygen has been studied from the point of view of infra-red emission. This has been rendered possible by the use of a Downing galvanometer kindly loaned to us by Prof. A. V. Hill. With this galvanometer and a Hilger infra-red spectrometer, it is a comparatively easy matter to survey the infra-red spectra of gaseous explosions. The explosion spectrum of carbon monoxide and oxygen is qualitatively the same as that of the flame, but the ratios of the intensities of the $2 \cdot 8 \mu$ and the $4.4 \mu$ bands are widely different in the two cases. For the explosion of the gases dried over $\mathrm{P}_{2} \mathrm{O}_{5}$ this ratio is nearly 1 : I for a $35 \mathrm{~cm}$. tube, and for the flame I : II. When the gases are saturated with water vapour the ratios are 2: I and I : Io respectively. The intensity of infra-red emission is markedly greater for the dry than for the wet gases, although the speed of explosion in the former is slower. The ratios of the deflexions obtained for the dry and wet gases were about $3: 2$ for the $2 \cdot 8 \mu$ band, and $3:$ I for the $4 \cdot 4 \mu$ band. The absorption of infra-red radiation by the water vapour in front of the explosion wave is negligible (cf. Hettner, Ann. Phys., I9I8, 55, 496). This we have checked by absorption measurements on the wet and dry explosive mixtures. Thus the decrease in the infra-red emission which occurs when water is added to the explosion of carbon monoxide must be ascribed to some cause operating on the hot gases themselves.

Two mechanisms may be put forward to explain the catalytic action of water which take into account the bearing of the decrease in the emission of infra-red radiation on the increase in the speed of reaction. The reduction in the intensity of the radiation may be due either to its absorption or to the prevention of its emission by the water molecules. In the latter case, the internal energy of those activated molecules whereas in the presence of an excess of iodine ions charged negatively. The potential difference between the solution and solid silver iodide varying with the

$$
\text { NO. 2953, VOL. I I 7] }
$$

